\title{
Integer points close to convex surfaces
}

\author{
by \\ M. C. Lettington (Cardiff)
}

1. Introduction. Let $C$ be the boundary surface of a strictly convex bounded three-dimensional body. Strictly convex means that if $P$ and $Q$ are points on $C$, then points on the line segment $P Q$ between $P$ and $Q$ lie in the convex body, but not on its boundary $C$. Let $M C$ denote the dilation of $C$ by a factor $M$. Andrews [1] proved a general result which in three dimensions gives the number of points of the integer lattice on $M C$ to be

$$
O\left(M^{3 / 2}\right),
$$

as $M$ tends to infinity. Strict convexity is necessary because a part of a two-dimensional plane in the boundary $C$ can give as many as a constant times $M^{2}$ integer points for infinitely many values of $M$.

We consider the integer points within a distance $\delta$ of the surface $M C$. The two-dimensional case has been well-studied ([12], [5], [9], [6], [10], [11]). Introducing $\delta$ requires some uniform approximability condition on the surface $C$, usually expressed in terms of upper and lower bounds for derivatives and determinants of derivatives. Let $A$ be the two-dimensional area of $C$. The search region has three-dimensional volume

$$
\left(2 A \delta+O\left(\delta^{2}\right)\right) M^{2}
$$

and this is known to be the number of integer points on average over translations of the surface $M C$. To obtain an asymptotic formula one considers the Fourier transform of the convex body, with conditions at least as far as the 18th derivatives in order to estimate the multiple exponential integrals [7]. Hlawka [8] obtained a general dimensional asymptotic formula, which in three dimensions yields an error of size (1); see also Krätzel [13]. Under the $C^{\infty}$ hypothesis of a convergent Taylor series, the error term in the asymptotic formula has been improved, most recently by Müller [18].

2000 Mathematics Subject Classification: Primary 11P21; Secondary 11H06, 11J25. Key words and phrases: lattice points, convex bodies. 
We derive an upper bound for the number of integer points within a distance $\delta$ of the surface. We require only that $C$ has a tangent plane at every point, and that any two-dimensional cross-section through the normal at some point $P$ consists (in a neighbourhood of $P$ ) of a plane curve $C^{\prime}$ with continuous radius of curvature $\varrho^{\prime}$ satisfying a condition

$$
1 / M<c_{0}<\varrho^{\prime}<c_{1} .
$$

This condition involves derivatives up to the second order. We actually suppose that $M$ is large and we assume the condition

$$
c_{0} M+1 / 2 \leq \varrho=\varrho^{\prime} M \leq c_{1} M-1 / 2,
$$

with $C$ contained in a sphere of radius $c_{1} M$.

Our upper bound has two terms whose orders of magnitude correspond to (1) and (2). The constant factor in the second term is larger than $2 A$.

In Müller [18] the differential inequality assumed is that the Gaussian curvature does not vanish. We can regard (3) as a corresponding quantitative bound.

Under the curvature conditions we prove that an upper bound for the number of integer points $N$ lying on or within a distance $\delta$ from the surface $C$ is given by

$$
N \leq\left(\frac{c_{1}}{c_{0}}\right)^{2} 2^{16}\left(\left(c_{1} M\right)^{3 / 2}+2^{9} \delta\left(c_{1} M\right)^{2}\right) .
$$

In Section 3 we consider the convex hull of the integer points in a $d$-dimensional convex body satisfying the Curvature Condition (introduced in Section 2), and find explicit bounds for the number of faces in different dimensions.

There is a vast literature on the lattice points in a convex polytope. We have been able to use some parts of this theory ([1], [2], [3], [4], [16], $[17],[19])$. Where possible, this theory has been stated in the general $d$ dimensional case. To extend all the results of this paper to $d$ dimensions requires more investigation of configurations in intermediate dimensions, and of the distribution of large faces of the convex hull with short normal vectors. This will be the subject of a following paper [15].

2. Shells and curvature. Let $C_{0}$ be the locus of points at distance $\delta$ from $C$ measured along the interior normals to $C$, and let $C_{1}$ be the locus of points at distance $\delta$ measured along the exterior normals. Let $E$ be the $d$-dimensional shell bounded by $C_{0}$ and $C_{1}$ so that $E$ has thickness $2 \delta$. Let $S$ be the set of integer points in $E$, and let $H$ be the convex hull of $S$, so that $H$ is a $d$-dimensional convex polytope. All points of $S$ lie in $H$, but not all integer points on the boundary of $H$ lie in $S$. 
Curvature Condition (with size parameter $M$ ). For any point $P$ on $C$ and any two-plane $\Pi$ through the normal to $C$ at $P$, let $C(\Pi, P)$ be the closed plane curve $C \cap \Pi$. Then $C(\Pi, P)$ is twice differentiable with radius of curvature $\varrho$ lying in the range

$$
c_{0} M+1 / 2 \leq \varrho \leq c_{1} M-1 / 2,
$$

where the constants $c_{0}, c_{1}$ and $\delta$ satisfy

$$
1 / M<c_{0} \leq 1 \leq c_{1}, \quad \delta<1 / 4 .
$$

As an immediate consequence of the Curvature Condition we have the following lemma.

Lemma 2.1. Let $C$ satisfy the Curvature Condition. For both of the boundary hypersurfaces $C_{0}$ and $C_{1}$ of the shell $E$, at each point $Q$ of the hypersurface there is a tangent hyperplane. The two-dimensional section $C(\Pi, Q)$ by a 2-plane normal to the tangent hyperplane is twice differentiable. The radius of curvature of $C(\Pi, Q)$ lies in the range

$$
c_{0} M \leq \varrho \leq c_{1} M .
$$

The proof follows by direct consideration of points on $C_{0}(\Pi, Q)$ and $C_{1}(\Pi, Q)$ with respect to $C(\Pi, Q)$.

By the condition (4), $S$, the set of integer points, lies in a $d$-hypersphere of radius $R=c_{1} M$, and we recall the formulae for $V_{d}$, the volume, and for $S_{d}$, the surface content, of a $d$-dimensional sphere [19]:

$$
V_{d}=\alpha_{d} R^{d}, \quad S_{d}=d \alpha_{d} R^{d-1},
$$

where

$$
\alpha_{2 k}=\frac{\pi^{k}}{k !}, \quad \alpha_{2 k+1}=\frac{2^{2 k+1} \pi^{k} k !}{(2 k+1) !}, \quad \alpha_{d} \leq 6 .
$$

3. Convex polytopes. In this section we again consider the general $d$-dimensional case, so that the convex hull $H$ of the set of integer points $S$ is a $d$-dimensional convex polytope, where $d \geq 2$.

Lemma 3.1. To each hypersurface face of the convex polytope $H$ we assign a standard normal vector; this is the unique outward normal integer vector $\left(A_{1}, \ldots, A_{d}\right)$, which is primitive in the sense that $\operatorname{hcf}\left(A_{1}, \ldots, A_{d}\right)=1$. Then for each $N \geq 1$ there are

$$
\leq 3^{d} N^{d}
$$

hyperfaces of $H$ whose standard normal vector has length at most $N$.

Proof. There are $2 N+1$ possibilities for each vector entry, so that the total possible number of vectors is

$$
(2 N+1)^{d} \leq 3^{d} N^{d}
$$


Lemma 3.2. Let $U$ be a set of $K$ integer points in d-dimensional space that do not all lie on some hyperplane. Then there is a simplicial complex of at least $K-d$ non-overlapping simplices whose vertices are the $K$ points of $U$.

Proof. This lemma may be proved from the outside in, by successively removing vertices of the convex hull of $U$, or from the inside out by choosing simplices of minimal non-zero volume [14].

Lemma 3.3. Let $H$ be a convex polytope contained in a hypersphere of radius $R$, whose vertices are integer points. Then the number of $(d-1)$ hyperplane faces of $H$ whose standard normal vector has length greater than $N$ is

$$
\leq \frac{\alpha_{d} R^{d-1} d !}{N}
$$

Proof. Consider $d$ integer points $\mathbf{x}_{1}, \ldots, \mathbf{x}_{d}$ lying on a hyperplane face with primitive normal vector $\left(A_{1}, \ldots, A_{d}\right)$, where the $d$-integer points form a simplex with $(d-1)$-dimensional volume $V^{(d-1)}$, and $\mathbf{x}_{d+1}$, an integer point lying off the hyperplane face. The perpendicular distance from $\mathbf{x}_{d+1}$ to the hyperplane face is

$$
D=\frac{k}{\sqrt{\left(A_{1}^{2}+\cdots+A_{d}^{2}\right)}}
$$

for some positive integer $k$. We chose $\mathbf{x}_{d+1}$ so that the distance is minimal and so $k=1$. Then the $d$-dimensional volume $V^{(d)}$ of the convex hull of these $d+1$ points satisfies

$$
V^{(d)}\left(\mathbf{x}_{1}, \ldots, \mathbf{x}_{d+1}\right)=\frac{1}{d} D V^{(d-1)}\left(\mathbf{x}_{1}, \ldots, \mathbf{x}_{d}\right) .
$$

Since the volume of a $d$-simplex whose vertices are integer points is at least $1 / d$ !, we have

$$
\begin{aligned}
V^{(d-1)}\left(\mathbf{x}_{1}, \ldots, \mathbf{x}_{d}\right) & \geq \frac{d}{(d) !} \frac{1}{D}=\frac{1}{(d-1) !} \sqrt{\left(A_{1}^{2}+\cdots+A_{d}^{2}\right)} \\
& \geq \frac{N}{(d-1) !}
\end{aligned}
$$

by the conditions of the lemma.

The $(d-1)$-dimensional hypervolume of the hyperplane faces of the convex polytope must be less than or equal to the $(d-1)$-hypervolume of the surface of the $d$-dimensional hypersphere enclosing it. Let $A_{i}$ be the hypervolume of each hyperplane face of the polytope; then by equation (7) we have

$$
\sum A_{i} \leq S_{d}=d \alpha_{d} R^{d-1}
$$


We obtain an upper bound for the number of large hyperplane faces of the convex polytope by dividing the lower bound (12) into the upper bound (13) to obtain

$$
\leq \frac{d \alpha_{d} R^{d-1}(d-1) !}{N} .
$$

TheOREM 3.4. Let $H$ be a convex polytope contained in a d-sphere of radius $R$. Then $H$ has at most

$$
2\left(3 \alpha_{d} d !\right)^{d /(d+1)} R^{d(d-1) /(d+1)}
$$

hyperplane faces.

Proof. We take

$$
N=\left(\frac{\alpha_{d} d !}{3^{d}}\right)^{1 /(d+1)} R^{(d-1) /(d+1)}
$$

in (9) of Lemma 3.1 and (10) of Lemma 3.3. The total number of hyperplane faces is the sum of bounds for those with long normal vectors in (9) and those with short normal vectors in (10), and is

$$
\leq \frac{\alpha_{d} R^{d-1} d !}{N}+(3 N)^{d}=2\left(3 \alpha_{d} d !\right)^{d /(d+1)} R^{d(d-1) /(d+1)} .
$$

LEMma 3.5. Let $H$ be a convex d-polytope with vertices at integer points. From each $j$-face $F_{i}$ of $H$, we pick out $j+1$ vertices $\mathbf{v}_{i, 1}, \ldots, \mathbf{v}_{i, j+1}$ that do not all lie on a $(j-1)$-dimensional plane. Let $\mathbf{w}_{i}$ be the centroid of these vertices:

$$
\mathbf{w}_{i}=\frac{1}{j+1}\left(\mathbf{v}_{i, 1}+\cdots+\mathbf{v}_{i, j+1}\right) .
$$

Let $T=\left\{\mathbf{w}_{1}, \ldots, \mathbf{w}_{h}\right\}$ be the set of centroids associated with all the $j$-faces of $H$. For a set $U$, let $\operatorname{conv}\{U\}$ denote the smallest convex set containing all the elements of $U$. Then the centroids $\mathbf{w}_{i}$ are true vertices of $\operatorname{conv}\{T\}$, in the sense that for any $t=1, \ldots, h$,

$$
\operatorname{conv}\left\{T \backslash\left\{\mathbf{w}_{h}\right\}\right\} \neq \operatorname{conv}\{T\} .
$$

Proof. We must rule out the possibility that

$$
\mathbf{w}_{i}=\sum_{g=1}^{h} \lambda_{g} \mathbf{w}_{g},
$$

with

$$
0 \leq \lambda_{g} \leq 1, \quad \sum_{g=1}^{h} \lambda_{g}=1
$$


Substituting for $\mathbf{w}_{g}$ using (16) and multiplying by $j+1$ to clear fractions yields

$$
\mathbf{v}_{i, 1}+\cdots+\mathbf{v}_{i, j+1}=\sum_{g=1}^{h} \sum_{f=1}^{j+1} \lambda_{g} \mathbf{v}_{g, f} .
$$

Each $j$-face $F_{i}$ is the intersection of at least $d-j$ facets or hyperplanes of $H$, and our $j+1$ vertices of $F_{i}$ are also vertices of each of these hyperplanes. We label these hyperplanes $\Pi_{1}, \ldots, \Pi_{k}, \ldots, \Pi_{t}$ with primitive integer normal vectors $\mathbf{n}_{k}$, so that any point $\mathbf{r}$ lying on $\Pi_{k}$ satisfies the equation

$$
\mathbf{r} \cdot \mathbf{n}_{k}=D_{k} \text {. }
$$

As $H$ is convex, all the $\Pi_{k}$ are supporting hyperplanes of $P$. Hence, for any point $\mathbf{x}$ in $H$ we have

$$
\mathbf{x} \cdot \mathbf{n}_{k} \leq D_{k},
$$

where we have assumed (using a suitable integer vector translation) that $H$ contains the origin. Applying (20) to (19) yields

$$
\begin{aligned}
\left(\mathbf{v}_{i, 1}+\cdots+\mathbf{v}_{i, j+1}\right) \cdot \mathbf{n}_{k} & =D_{k}(j+1)=\sum_{g=1}^{h} \sum_{f=1}^{j+1} \lambda_{g} \mathbf{v}_{g, f} \cdot \mathbf{n}_{k} \\
& \leq(j+1) \sum_{g=1}^{h} \lambda_{g} D_{k}=D_{k}(j+1),
\end{aligned}
$$

implying that

$$
D_{k}(j+1)=\sum_{g=1}^{h} \lambda_{g} \sum_{f=1}^{j+1} \mathbf{v}_{g, f} \cdot \mathbf{n}_{k} \leq D_{k}(j+1) .
$$

This equality is only satisfied if all of the vertices $\mathbf{v}_{g, f}$ for which $\lambda_{g} \neq 0$ are on the hyperplanes $\Pi_{k}, 1 \leq k \leq t$.

Now any $j$-face $F_{i}$ of a convex $d$-polytope $H$ can be defined as the intersection of the $q$-faces that contain $F_{i}$ with $j \leq q \leq d-1$. Therefore, as the vertices $\mathbf{v}_{g, f}$ lie on such an intersection with $q=d-1$, we deduce that the vertices $\mathbf{v}_{g, f}$ for which $\lambda_{g} \neq 0$ are all vertices of our $j$-face $F_{i}$. That is, $\mathbf{v}_{g, 1}, \ldots, \mathbf{v}_{g, j+1}$ are vertices of $F_{i}$.

This implies that for $g \neq i$ in equation (19) we must have $\lambda_{g}=0$, as two distinct $j$-faces of $H$ cannot share $j+1$ vertices. Hence there is only one term, $\lambda_{g}$, with $g=i$ and $\lambda_{i}=1$ yielding the trivial expression, right hand side is identical to left hand side in (19).

Therefore, $\mathbf{w}_{i}$ has only one expression as a convex sum of

$$
T=\left\{\mathbf{w}_{1}, \ldots, \mathbf{w}_{h}\right\},
$$

and thus $\mathbf{w}_{i}$ is not in the convex hull of $T-\mathbf{w}_{i}$. 
Theorem 3.6 is a version of Andrew's Theorem [1] with explicit constants. The second statement regarding the number of faces was not stated in [1]. McMullen [16] has upper bounds for the number of faces in terms of the vertices. These bounds can be attained by polytopes with integer vertices lying on a twisted quantic curve, but the parameter $R$ is very large. Hence, for a spherically contained convex $d$-polytope, there exist triples, $\left(d, f_{0}, j\right)$, for which the second statement of Theorem 3.6 is an improvement on the general upper bound stated by McMullen in [16].

Theorem 3.6. In d-dimensional space, a convex polytope $H$ with $f_{0}$ vertices, all at integer points, contained in a hypersphere of radius $R$ satisfies

$$
f_{0} \leq 2\left(3 \alpha_{d} d !\right)^{d /(d+1)}(2 R)^{d(d-1) /(d+1)} \leq 36 d !(2 R)^{d(d-1) /(d+1)} .
$$

Let $1 \leq j \leq d-2$. Under the conditions of the theorem, the number $f_{j}$ of $j$-faces of $P$ satisfies

$$
f_{j} \leq 2\left(3 \alpha_{d} d !\right)^{d /(d+1)}(2(j+1) R)^{d(d-1) /(d+1)} .
$$

Proof. Let $T$ be the set of midpoints of edges of $H$, and let $H^{\prime}$ be the convex hull of $T$. By Lemma 3.5 each point of $T$ is a vertex of $H^{\prime}$. Let $V$ be the vertex of $H$ where edges $e_{1}, \ldots, e_{r}$ meet and let $W_{1}, \ldots, W_{r}$ be the respective midpoints of these edges. The $W_{1}, \ldots, W_{r}$ are all vertices of $H^{\prime}$ but not necessarily of the same facet.

By construction, each vertex $V$ of $H$ is truncated by a facet $F$ of $H^{\prime}$ and we say that $V$ belongs to the facet $F$. Geometrically we can think of $V$ as lying above the facet $F$. The supporting hyperplane $\Pi$ of $H^{\prime}$ containing $F$ cuts $H$ in a $(d-1)$-dimensional convex polytope $Q$. The join of $V$ to any other vertex $V^{\prime}$ of $H$ cuts $\Pi$ within this convex polytope. We now show that $V^{\prime}$ cannot lie above the facet $F$. The vertices of $Q$ are points $X_{1}, \ldots, X_{r}$ on $e_{1}, \ldots, e_{r}$ and $X_{i}$ is either $W_{i}$, the midpoint of $e_{i}$, or between $V$ and $W_{i}$. Therefore, if $V^{\prime}$ lies above $F$, then $V^{\prime}$ lies in $\operatorname{conv}(Q, V)$ and so $V^{\prime}$ lies in $\operatorname{conv}\left(V, X_{1}, \ldots, X_{r}\right)$. The only vertex of $H$ in this list is $V$, so $V^{\prime}=V$.

This implies that the number of facets of $H^{\prime}$ is greater than or equal to the number of vertices of $H$.

Now $2 H^{\prime}$ is a polytope with integer vertices lying in a $d$-sphere of radius $2 R$, so the number of faces of $H^{\prime}$ is given by (15) of Theorem 3.4, but with a larger implied constant. We deduce the result (22).

For each $j$-face $G$ of $H$ we choose $j+1$ vertices that do not all lie on the same $(j-1)$-plane and construct $C(G)$, the centroid of the $j+1$ vertices. Since $C(G)$ does not lie on the $(j-1)$-dimensional boundary of $G$, we see that $C(G)$ cannot lie on any other $j$-face. Let $U$ be the set of centroids $C(G)$ constructed from the $j$-faces of $H$.

By Lemma 3.5, $U$ is a strictly convex set and we define $H^{\prime \prime}$ to be the convex hull of the points $C(G)$ in $U$. Then $(j+1) H^{\prime \prime}$ is a polytope with 
integer point vertices lying in a sphere of radius $(j+1) R$, so that the number of vertices of $H^{\prime \prime}$ is given by equation (22), but with a larger implied constant. Each $j$-face $G$ gives a distinct point $C(G)$ in $U$ which is a vertex of the convex polytope $H^{\prime \prime}$. We deduce the result (23).

4. Major arcs and lattices. Throughout this section $H$ is the convex hull of the set $S$ of integer points inside the shell $E$. It is helpful in many problems to separate "major arcs", regions where there is good Diophantine approximation, from "minor arcs", regions where there is not. In this paper a major arc can be described informally as a region $U$ of the shell $E$ such that the convex hull of all the integer points in $U$ is contained in the intersection of $E$ with some hyperplane. In three dimensions, major arcs on the plane faces and edges of the convex hull $H$ can have dimension 1 or 2 .

LEMMA 4.1. The maximum length of a straight line segment in $E$ is

$$
\leq 4 \sqrt{\delta c_{1} M} .
$$

$A$ chord $A B$ of $C_{1}$ tangent to $C_{0}$ has length

$$
4 \sqrt{\delta c_{0} M} \leq A B \leq 4 \sqrt{\delta c_{1} M} .
$$

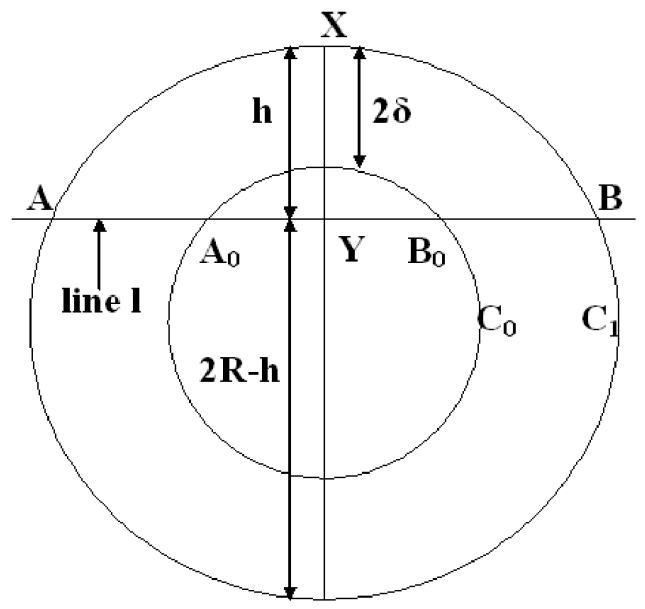

Fig. 1. Section by 2-plane $\Pi$ through $l$ and $X$

Proof. Let $R=c_{1} M$ and let $\Pi$ be a two-dimensional plane containing the normal vector at a point $X$ on $C_{1}$ and any other point $A$ also on the outer boundary $C_{1}$. The two-dimensional section $E^{\star}$ of the shell $E$ in $\Pi$ is depicted in Figure 1. We consider the line segment $A B$ when it is wholly contained within $E^{\star}$ and so the perpendicular distance $X Y$ from $A B$ to $X$ must be $\leq 2 \delta$. Applying circular geometry to the circle of radius $R$ with 
respect to the mid-point $Y$ of chord $A B$ we find that

$$
\left(\frac{A B}{2}\right)^{2}=A Y^{2}=X Y(2 R-X Y),
$$

and for fixed $R$, this is maximal when $X Y=R$. Hence we take $X Y$ as large as possible in (26), yielding the required result.

The lower bound in (25) corresponds to the case when the cross-sectional curve is a circle of minimal radius of curvature $R=c_{0} M+1 / 2$. In this case, by $(26)$,

$$
A B=2 A Y=4 \sqrt{\delta(R-\delta)} \geq 4 \sqrt{\delta c_{0} M},
$$

by (4) and (5).

Lemma 4.2. Let $R=c_{1} M$ and let $F$ be a facet or hyperplane face of $H$ that lies in a hyperplane $\Psi$ with outward normal $\mathbf{n}$. Let $X$ be the point of $C_{1}$ at which $\mathbf{n}$ is the outward normal. Let $h$ be the distance from $X$ along the inward normal to the nearest point $Y$ on the hyperplane $\Psi$. Let $E^{\prime}$ be the $(d-1)$-dimensional section of $E$ contained in $\Psi$, so that $E^{\prime}$ contains all parts of the face $F$ that lie in the shell $E$. Then the $(d-1)$-dimensional volume $V$ of $E^{\prime}$ is bounded above by

$$
V \leq 2^{(d+9) / 2} d \delta R^{(d-1) / 2} h^{(d-3) / 2} .
$$

Proof. Let $\Pi$ be a two-dimensional plane through $X Y$, and let $E^{\star}$ be the two-dimensional section of $E$ by $\Pi$ (Figure 1). Then $\Pi$ cuts $\Psi$ in a straight line $l$ which meets $C_{1}$ in two distinct points $A$ and $B$. The points $A$ and $B$ lie inside the circle of radius $R$ through $X$ with $\mathbf{n}$ as outward normal at $X$. For clarity, the curves $C_{0}$ and $C_{1}$ in Figure 1 are drawn as circles. From (26) in the proof of Lemma 4.1 we have

$$
A Y \leq \sqrt{h(2 R-h)}=k .
$$

Hence the set $E^{\prime}=E \cap \Psi$ lies within a $(d-1)$-sphere with centre $Y$ and radius $\leq \sqrt{2 R h}$.

CASE 1 . When $h \leq 2 \delta$, the plane $\Psi$ does not cut $C_{0}$ and, by (6), the diameter of $E^{\prime}$ satisfies (24). This implies that the whole of the facet $F$ is contained within the shell $E$. Therefore, the $(d-1)$-dimensional volume $V$ of $E^{\prime}$ is less than or equal to that of a $(d-1)$-sphere of radius $\sqrt{2 h R}$. Applying (7) yields

$$
V \leq \alpha_{d-1}(2 h R)^{(d-1) / 2} \leq 2^{(d+5) / 2}(h R)^{(d-1) / 2} .
$$

CASE 2. When $h>2 \delta$, the hyperplane $\Psi$ meets $C_{0}$, and the line $l$ in the two-dimensional plane $\Pi$ cuts $C_{0}$ in two distinct points $A_{0}$ and $B_{0}$. Let $A_{0} T$ be the normal from $A_{0}$ to $C_{1}$, so the distance $A_{0} T$ is $2 \delta$, and let $C^{\star}$ be the hypersphere of radius $R$ touching $C_{1}$ at $T$. Let $\Pi_{1}$ be the two-dimensional 


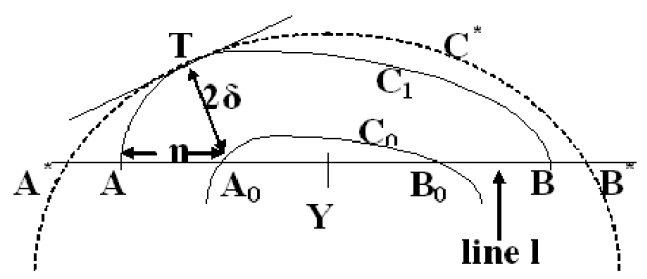

Fig. 2. Section by 2-plane $\Pi_{1}$ through $l$ and $T$

plane through the line $l$ and the point $T$ (Figure 2). Then $C_{1}$ and the shell $E$ are contained within $C^{\star}$. The line $l$ cuts $C^{\star}$ at $A^{\star}$ and $B^{\star}$, so that by the geometry of circles

$$
A A_{0} \cdot A_{0} B \leq A^{\star} A_{0} \cdot A_{0} B^{\star}=2 \delta(2 R-2 \delta) \leq 4 \delta R .
$$

On the line $l$, the point $A$ lies between $A^{\star}$ and $A_{0}$, with $A A_{0}=\eta$ (say) and $\eta>0$. Hence

$$
\eta \leq A^{\star} A_{0} .
$$

We also have

$$
A_{0} B^{\star} \geq Y B^{\star}=k=\sqrt{h(2 R-h)} .
$$

Each point of $E^{\prime}$ lies within a distance $\eta$ of the $(d-2)$-dimensional surface of $C_{1} \cap \Psi$. The $(d-2)$-dimensional volume of $C_{1} \cap \Psi$ is at most the surface content of a $(d-1)$-dimensional sphere of radius $k$, which by $(7)$ is equal to

$$
(d-1) \alpha_{d-1} k^{d-2} \text {. }
$$

Therefore, the $(d-1)$-dimensional volume $V$ of $E^{\prime}$ satisfies

$$
V \leq(d-1) \alpha_{d-1} \eta k^{d-2} .
$$

From (30)-(32) we have

$$
\eta k \leq A^{\star} A_{0} \cdot A_{0} B^{\star} \leq 4 \delta R .
$$

Hence we can write

$$
V \leq(d-1) \alpha_{d-1}(4 \delta R) k^{d-3},
$$

which simplifies to

$$
V \leq 2^{(d+7) / 2}(d-1) \delta R^{(d-1) / 2} h^{(d-3) / 2} .
$$

Combining (29) and (35) yields

$$
V \leq 2^{(d+9) / 2} d \delta R^{(d-1) / 2} h^{(d-3) / 2}
$$

and hence the result.

LEMMA 4.3. In d-dimensional space, the number of integer points of $S$ in $E$ that lie strictly inside the convex hull $H$ of $S$ is

$$
\leq 2 \delta d ! \alpha_{d} d\left(c_{1} M\right)^{d-1} .
$$


In particular, if $d=3$, then the number of integer points lying within a short distance $\delta$ of the convex hull $H$ is

$$
\leq 48 \pi \delta\left(c_{1} M\right)^{2} .
$$

Proof. Given that the integer point vertices of our convex hull $H$ lie within a distance $\delta$ from the closed convex hypersurface $C$, we can associate a hyperslab of width $2 \delta$ with each facet of the polytopal convex hull where the hyperslabs will overlap.

Any integer points $H \cap E$ must lie within a distance $2 \delta$ of the nearest polytope facet $F_{i}$ with hypersurface area $A_{i}$. The internal or "dihedral" angles between facets are $\leq 180^{\circ}$ due to convexity. Let $P$ be such a point with nearest hyperface $F_{i}$, so that the perpendicular from $P$ to the hyperplane $F_{i}$ actually hits $F_{i}$. If not, then some other hyperplane is nearer $\left(F_{j}\right.$ say) under the distance equation (11) defined in Lemma 3.3.

Therefore each integer point $P$ lying inside the convex hull can be associated uniquely with a nearest hyperface $F_{i}$.

Corresponding to each hyperface $F_{i}$ we have a hyperslab $S_{i}$ consisting of two completely parallel hyperfaces $F_{i}$ and $F_{i}$ shifted by $2 \delta$ in the normal direction to the hyperplane. The hypervolume of $S_{i}$ equals $2 \delta A_{i}$ where $A_{i}$ is the hypersurface area of $F_{i}$.

We know from Lemma 3.2 that in $d$-dimensions, $K$ points that do not all lie on the same hyperplane form at least $K-d$ non-overlapping simplices. Each simplex has hypervolume $1 / d$ ! multiplied by an integer so that each of these simplices has hypervolume $\geq 1 / d$ !

Therefore, if $K_{i}$ is the number of internal integer points associated uniquely with the hyperface $F_{i}$, which itself has at least $d$ integer point vertices, then the total number of internal and boundary integer points of the hyperface is

$$
\geq d+K_{i},
$$

so that we have at least $K_{i}$ non-overlapping simplicies, yielding

$$
\frac{K_{i}}{d !} \leq 2 \delta A_{i},
$$

which implies

$$
K_{i} \leq 2 d ! \delta A_{i} .
$$

Hence the total number of integer points lying within a short distance $\delta$ of the convex hull $H$ is

$$
\leq \sum_{i} K_{i} \leq \sum_{i} 2 d ! \delta A_{i} .
$$

The boundary content of our convex $d$-polytope $H$ is less than or equal to 
that of the hypersphere with radius of curvature $c_{1} M$ enclosing it. Therefore

$$
\sum_{i} K_{i} \leq 2 d ! \delta \alpha_{d} d\left(c_{1} M\right)^{d-1} .
$$

The next two lemmas are commonplaces of integer geometry, so we merely sketch the proofs.

LEMma 4.4. Let $\Pi$ be a hyperplane with equation

$$
\mathbf{n} \cdot \mathbf{x}=D,
$$

where $\mathbf{n}$ is a primitive integer vector, and $D$ is an integer. Then the integer points of $\Pi$ form a lattice with determinant $|\mathbf{n}|$.

Proof. The lattice of integers on $\Pi$ is congruent to the lattice of integers on the plane $\mathbf{n} \cdot \mathbf{x}=0$. Let $m=\mathbf{n} \cdot \mathbf{n}$. The lattice of integer vectors with $\mathbf{n} \cdot \mathbf{x} \equiv 0(\bmod m)$ consists of $(d-1)$-dimensional lattices on the plane $\mathbf{n} \cdot \mathbf{x}=0$ and on parallel planes at distance $|\mathbf{n}|$.

Lemma 4.5. Let $\Lambda$ be a $j$-dimensional lattice of determinant $n, 1 \leq j \leq d$. Let $U$ be a convex set in the $j$-plane of $\Lambda$, with $j$-dimensional volume $V$, containing $K$ points of the lattice $\Lambda$. Then one of the following two cases holds:

(1) Major arc case: All the points of $\Lambda$ in the set $U$ lie on $a(j-1)$ dimensional plane.

(2) Minor arc case:

$$
K \leq j ! \frac{V}{n}+j \leq(j+1) ! \frac{V}{n} .
$$

Proof. In the minor arc case, by Lemma 3.2, there is a simplicial complex of at least $K-j$ non-overlapping simplices, each of volume at least $n / j$ !, whose vertices are the $K$ points of $U$. The union of these simplices lies inside $U$ and this gives the first inequality. There is at least one such simplex, so $V \geq n / j$ !, and we deduce the second inequality.

5. Vertex components. From this point on, we are restricted to three dimensions.

For each point $P$ in our shell $E$, there exists a normal to the outer boundary surface $C_{1}$, meeting $C_{1}$ at a point $R_{1}$. We call $R_{1}$ the normal projection of $P$ onto $C_{1}$ and $R_{0}$ the normal projection of $P$ onto $C_{0}$. The vertices of our polyhedron must, by definition, lie in $E$, and for every other non-vertex integer point in $E$ there must exist a nearest vertex. We now formalise this concept with the following definition.

Definition. Let $P$ be a point of $S$ in the shell $E$ and $R_{1}$ be the normal projection of $P$ onto $C_{1}$. Let $V$ be a vertex of the convex polyhedral hull $H$ and $E^{\prime}$ be the plane sectional strip of $E$ containing $V, P$ and $R_{1}$. If the line 
segment $R_{1} V$ lies entirely within the closed strip $E^{\prime}$, then we say that $P$ lies in the component $S(V)$ of $S$.

LEMma 5.1. Every point $P$ of $S$ belongs to some vertex component $S(V)$.

Proof. The line segment $P R_{1}$ cuts the boundary of the polyhedral hull $H$ at some point $Q$ between $P$ and $R_{1}$ inside $E$, so that $Q$ lies in some plane face $F$ of $H$. If $Q$ is a vertex of $H$ then $P$ belongs to $S(Q)$ as $Q R_{1}$ will lie on the line segment $R_{0} R_{1}$ inside $E$.

We now assume that $Q$ is not a vertex of $H$ and triangulate the face $F$ of $H$ containing $Q$ so that $Q$ lies in some triangle $W=V_{1} V_{2} V_{3}$. If $Q V_{i}$ does not enter the interior of the convex set bounded by $C_{0}$ then neither does $R_{1} V_{i}$, implying that $P$ lies in $S\left(V_{i}\right)$.

If $P$ lies in no $S\left(V_{i}\right)$ then each line segment $Q V_{i}$ on $F$ cuts the interior of $C_{0}$ in some point $Q_{i}$ also on $F$ but not in $E$. The whole convex triangle $Q_{1} Q_{2} Q_{3}$ therefore lies strictly inside $C_{0}$ and contains $Q$. Hence, $Q$ is not in $E$, which is impossible, since $Q$ lies on the line segment $R_{0} R_{1}$, which is strictly inside $E$. This contradiction shows that for some $i$, the line segment $V_{i} Q$ lies in $E$ and so $V_{i} R_{1}$ lies in $E$ and $P$ is in the component corresponding to $V_{i}$.

Lemma 5.2 (Spacing lemma). Let $V$ be a vertex of the convex hull $H$. Let $P$ be a point of $S$ not in the component $S(V)$ of $V$. Let $R_{1}$ and $R_{2}$ be the respective normal projections of $P$ and $V$ onto $C_{1}$. Then

$$
R_{1} R_{2}>\sqrt{c_{0} \delta M}
$$

and the angle between the normals to $C_{1}$ at $R_{1}$ and $R_{2}$ is

$$
>\frac{1}{c_{1}} \sqrt{\frac{c_{0} \delta}{M}}
$$

Proof. Since $P$ is not in the component of $V$, the line $R_{1} V$ cuts $C_{0}$ in two points $W_{1}$ and $W_{2}$. Let $E^{\prime}$ be the plane sectional closed strip of $E$ defined by the line $R_{1} V$ and the point $R_{2}$, so that $E^{\prime}$ also contains the points $W_{1}$ and $W_{2}$. Between $W_{1}$ and $W_{2}$ on $C_{0}$ is a point $W$ where the tangent to $C_{0}$ in $E^{\prime}$ passes through $R_{1}$. Then

$$
R_{1} V>R_{1} W_{2}>R_{1} W \geq 2 \sqrt{\delta c_{0} M}
$$

by (25). Hence, by (4) and (5),

$$
R_{1} R_{2} \geq R_{1} V-2 \delta>2 \sqrt{\delta c_{0} M}-2 \delta \geq 2 \sqrt{\delta c_{0} M}-\sqrt{\delta c_{0} M}=\sqrt{\delta c_{0} M},
$$

which is (38).

To obtain (39) we consider the sphere $B$ with centre on $R_{2} V$, and radius $c_{1} M$, touching $C_{1}$ at $R_{2}$. There is a point $R_{1}^{\prime}$ on $B$ where the outward normal is parallel to the outward normal to $C_{1}$ at $R_{1}$, making some angle $\theta$ with 
the outward normal at $R_{2}$. Since $C_{1}$ has sectional radius of curvature less than or equal to $c_{1} M$, the radius of $B$, we have

$$
R_{1} R_{2} \leq R_{1}^{\prime} R_{2} .
$$

The shortest distance from $R_{1}^{\prime}$ to $R_{2}$ along the surface of $B$ is $\theta c_{1} M$, so

$$
\theta c_{1} M \geq R_{1}^{\prime} R_{2} \geq R_{1} R_{2}>\sqrt{c_{0} \delta M}, \quad \text { that is, } \theta>\frac{1}{c_{1}} \sqrt{\frac{c_{0} \delta}{M}},
$$

as required.

As each integer point $P$ in $S$ belongs to at least one component $S(V)$ labelled by some vertex $V$ of the convex hull $H$, components labelled by different vertices may well overlap and different vertices of the convex hull may be close together. We pick a well-spaced set of vertices of $H$ as follows. Pick a vertex $V_{1}$, and let the enlarged component $S^{\prime}\left(V_{1}\right)$ be the union of all components $S(V)$ with $V$ in $S\left(V_{1}\right)$.

Now pick a vertex $V_{2}$ not in $S^{\prime}\left(V_{1}\right)$, and form the enlarged component $S^{\prime}\left(V_{2}\right)$. We pick $V_{i+1}$ not in $S^{\prime}\left(V_{1}\right), \ldots, S^{\prime}\left(V_{i}\right)$, and so on until all of the vertices $V$ of the convex hull $H$ lie in some enlarged component.

Lemma 5.3 (Thickness lemma). Let $S^{\prime}(V)$ be an enlarged component and let $R_{2}$ be the normal projection of $V$ onto $C_{1}$. Let $P$ be a point in $S^{\prime}(V)$. Then the distance $h$ of $P$ from the tangent plane at $R_{2}$ satisfies

$$
h \leq \frac{52 \delta c_{1}}{c_{0}} .
$$

Proof. The integer point $P$ lies in some component $S\left(V^{\prime}\right)$ with $V^{\prime}$ in $S^{\prime}(V)$. Let $R_{1}$ and $R_{2}^{\prime}$ be the respective normal projections of $P$ and $V^{\prime}$ onto $C_{1}$. The line segments $R_{1} V^{\prime}$ and $R_{2}^{\prime} V$ lie inside the shell $E$, so by Lemma 4.1,

$$
R_{1} V^{\prime} \leq 4 \sqrt{\delta c_{1} M}, \quad R_{2}^{\prime} V \leq 4 \sqrt{\delta c_{1} M} .
$$

The distances $V^{\prime} R_{2}^{\prime}$ and $V R_{2}$ are at most $2 \delta$, so

$$
R_{1} R_{2} \leq R_{1} V^{\prime}+V^{\prime} R_{2}^{\prime}+R_{2}^{\prime} V+V R_{2} \leq 8 \sqrt{\delta c_{1} M}+4 \delta \leq 10 \sqrt{\delta c_{1} M},
$$

where we have used (4) and (5).

Let $E^{\prime}$ be the plane sectional strip of $E$ defined by $R_{1}, V$ and the normal projection $R_{2}$ of $V$ onto $C_{1}$. Let $C^{\prime}$ be the convex curve defined by the intersection of $C_{1}$ and $E^{\prime}$.

For fixed distance $R_{1} R_{2}=D$, the distance of $R_{1}$ from the tangent to $C^{\prime}$ at $R_{2}$ in $E^{\prime}$ is greatest when the radius of curvature is least, which is when $C^{\prime}$ is an arc of a circle of radius $c_{0} M$. Let $\alpha$ be the angle between $R_{1} R_{2}$ and the tangent at $R_{2}$. In the extreme case when $C^{\prime}$ is a circle of radius $c_{0} M$, the chord $R_{1} R_{2}$ subtends an angle $2 \alpha$ at the centre of the circle, so

$$
D=2 c_{0} M \sin \alpha,
$$


and the distance of $R_{1}$ from the tangent at $R_{2}$ is

$$
D \sin \alpha=\frac{D^{2}}{2 c_{0} M} \leq \frac{100 \delta c_{1} M}{2 c_{0} M}=\frac{50 \delta c_{1}}{c_{0}} .
$$

The distance of $P$ from the tangent plane to $C_{1}$ at $R_{2}$ is therefore

$$
\leq \frac{50 \delta c_{1}}{c_{0}}+2 \delta \leq \frac{52 \delta c_{1}}{c_{0}}
$$

REMARK. If we can obtain a bound valid for $\delta$ sufficiently small, then we can deduce a possible weaker bound for large $\delta$ by dividing the shell $E$ into concentric shells $E_{r}, 1 \leq r \leq R$, of thickness $\delta_{0}$, bounded by shrunken copies of the exterior surface $C_{1}$ of $E$. By inequality (6), we have a uniform upper bound of $c_{1} M$ for the sectional radius of curvature at any point on each shell $E_{r}$. Hence, when regarding maximum sectional radius of curvature, we can work within the general shell boundary $C_{1}$, whose sectional radius of curvature is also $\leq c_{1} M$.

Lemma 5.4 (Flatness lemma). Let $S^{\prime}(V)$ be an enlarged vertex component of our convex polyhedral hull $H$. If

$$
\delta<\delta_{0}=\sqrt{\frac{c_{0}}{2^{7} 3 \cdot 5^{2} 13 c_{1}}} \cdot \frac{1}{\sqrt{c_{1} M}},
$$

then all the points of $S^{\prime}(V)$ lie on a plane through the vertex $V$.

Proof. Let $R_{2}$ be the normal projection of $V$ onto $C_{1}$. All points $P$ of $S^{\prime}(V)$ lie within a distance $52 \delta c_{1} / c_{0}$ from the tangent plane at $R_{2}$, and by $(41)$,

$$
P V \leq P R_{1}+R_{1} V^{\prime}+V^{\prime} R_{2}+R_{2} V \leq 8 \sqrt{\delta c_{1} M}+4 \delta \leq 10 \sqrt{\delta c_{1} M} .
$$

Hence, the set $S^{\prime}(V)$ of integer points lies within a rectangular box $L$, of volume $\operatorname{Vol}(L)$, with

$$
\operatorname{Vol}(L) \leq \frac{52 \delta c_{1}}{c_{0}}\left(20 \sqrt{\delta c_{1} M}\right)^{2}<\frac{1}{6},
$$

where we have used the assumption (42). Therefore, by Lemma 4.5 the major arc case holds, and all points of the enlarged vertex component $S^{\prime}(V)$, including $V$ itself, lie on a plane.

Lemma 5.5 (Approximate tangency). Let $S^{\prime}(V)$ be an enlarged component. Let $T$ be the point of $C_{1}$ closest to $V$. Let $P$ be another point of $S^{\prime}(V)$, and let $\mathbf{g}$ be the integer vector $V P$. Then the angle $\alpha$ between $V P$ and the normal to $C_{1}$ at $T$ satisfies

$$
|\cos \alpha| \leq \frac{52 \delta c_{1}}{c_{0}|\mathbf{g}|}
$$

Proof. Let $\Pi$ be the plane through $P$ and the normal to $C_{1}$ at $T$ through $V$. Then $C_{1}$ will appear in $\Pi$ as a convex curve $C^{\prime}$. Let $l$ be the tangent to $C^{\prime}$ 
at $T$, and let $U$ be the foot of the perpendicular from $P$ to $l$ in $\Pi$. If $W$ is the foot of the perpendicular from $V$ to $P U$ then $V T U W$ is a rectangle in $\Pi$.

By Lemma 5.3 we have

$$
P U \leq 52 \delta c_{1} / c_{0} .
$$

Now if $P$ is between $W$ and $U$, then

$$
V P|\cos \alpha|=P W \leq W U=V T \leq 2 \delta,
$$

and if $W$ is between $P$ and $U$ then

$$
V P|\cos \alpha|=P W \leq P U \leq 52 \delta c_{1} / c_{0} .
$$

The inequality (44) holds in both cases.

Lemma 5.6 (Sums of reciprocals). We have

$$
\sum_{1 \leq|\mathbf{e}| \leq E} \frac{1}{|\mathbf{e}|} \leq 2^{6} E^{2}
$$

Proof. Applying the Cauchy condensation method, we divide the normal vectors into ranges

$$
F / 2<|\mathbf{e}| \leq F, \quad F=1,2,4, \ldots, 2^{K},
$$

where $2^{K}$ is the largest power of 2 less than or equal to $E$. The number of integer vectors in this range is

$$
\leq(2 F+1)^{3}-(F+1)^{3} \leq 19 F^{3}
$$

so that

$$
\sum_{F / 2<|\mathbf{e}| \leq F} \frac{1}{|\mathbf{e}|} \leq 19 F^{3} \cdot \frac{2}{F}=38 F^{2} .
$$

Summing over the ranges for $F$, we have

$$
\sum \frac{1}{|\mathbf{e}|} \leq 38\left(1+4+16+\cdots+2^{2 K}\right) \leq \frac{39\left(2^{2 K+2}-1\right)}{4-1} \leq 13 \cdot 4\left(2^{2 K}\right) \leq 2^{6} E^{2} .
$$

6. Plane faces and edges. In Lemma 4.3, we counted all of the integer points in the extended vertex components that lie strictly inside the convex hull $H$. Therefore we need only consider the set $S(H)$ of integer points in our extended vertex components that lie strictly on the plane faces and edges of $H$ in $S$.

Let $S^{\star}\left(V_{i}\right)$ be the subset of $S^{\prime}\left(V_{i}\right)$ consisting of integer points on the boundary of $H$. We will call this a boundary component. We have shown that for each extended vertex component $S^{\prime}\left(V_{i}\right)$, if $\delta$ is sufficiently small then $S^{\prime}\left(V_{i}\right)$ lies in a plane and so $S^{\star}\left(V_{i}\right)$ lies in the same plane. 
LEMma 6.1. The number of integer points on one-dimensional boundary components is estimated by

$$
\sum_{\operatorname{dim} S^{\star}\left(V_{i}\right)=1}\left|S^{\star}\left(V_{i}\right)\right| \leq \frac{2^{16} 3^{3} \pi c_{1}}{c_{0}^{2}} \delta\left(c_{1} M\right)^{2} .
$$

Proof. First we note that at most two one-dimensional components can lie on the same straight line. If this were not the case and there were more than two, then there would exist at least two seperate sections of a straight line segment that do not lie in the shell $E$. This in turn means that there exists a straight line segment that cuts $C_{0}$ or $C_{1}$ more than twice, which contradicts the convexity property assumed.

We consider all the boundary components $S^{\star}\left(V_{i}\right)$ which are one-dimensional lying parallel to some primitive integer vector e. Suppose that the component contains $l$ points of $S(H)$, where

$$
L+1 \leq l \leq 2 L
$$

for some $L$ equal to a power of two. We can take $\mathbf{g}=(l-1) \mathbf{e}$ in Lemma 5.5, with

$$
|\mathbf{g}| \geq(l-1)|\mathbf{e}| \geq L|\mathbf{e}| .
$$

In Lemma 5.5 the angle $\alpha$ between the vector $\mathbf{e}$ and the normal to $C_{1}$ at $T$, the point of $C_{1}$ nearest to $V$, satisfies

$$
|\cos \alpha| \leq \frac{52 \delta c_{1}}{c_{0} L|\mathbf{e}|}
$$

Hence

$$
\left|\frac{\pi}{2}-\alpha\right| \leq \frac{26 c_{1} \pi \delta}{c_{0} L|\mathbf{e}|}
$$

We want to discuss the spacing of the vertices $V_{i}$ that label the enlarged components $S^{\prime}\left(V_{i}\right)$ and so the boundary components $S^{\star}\left(V_{i}\right)$. Each $V_{i}$ has a normal projection $T_{i}$ on $C_{1}$. Consider a sphere $B$ of radius $c_{1} M$. We map $T_{i}$ on $C_{1}$ to the point $W_{i}$ on $B$ where the outward normal $\mathbf{n}$ to $B$ is parallel to the outward normal to $C_{1}$ at $T_{i}$.

Let $V_{i}$ and $V_{j}$ be distinct vertices labelling enlarged vertex components. Since $V_{j} \notin S\left(V_{i}\right)$, we have

$$
T_{i} T_{j}>\sqrt{c_{0} \delta M}
$$

by (38) of Lemma 5.2. Since $C_{1}$ has sectional radii of curvature at most $c_{1} M$,

$$
W_{i} W_{j} \geq T_{i} T_{j}>\sqrt{c_{0} \delta M} .
$$

Hence balls $B_{i}$ of radii $\frac{1}{2} \sqrt{c_{0} \delta M}$ centred at the points $W_{i}$ on $B$ are disjoint.

The ball $B_{i}$ intersects with the surface of the sphere $B$ in a set $A_{i}$ which contains the centre $W_{i}$ of $B_{i}$ and is a two-dimensional ball in spherical 
geometry. As the $B_{i}$ are disjoint, the areas $A_{i}$, on the surface of the sphere $B$ are also disjoint and do not overlap. Hence different sets $S^{\prime}\left(V_{i}\right)$ correspond to disjoint sets $A_{i}$, centred at $W_{i}$, on the surface of the sphere $B$. The area of $A_{i}$ is greater than the area of the intersection of a plane through $W_{i}$ with $B_{i}$, which is

$$
\pi c_{0} \delta M / 4
$$

As $V_{i} \in S^{\star}\left(V_{i}\right)$ and $S^{\star}\left(V_{i}\right) \subseteq S^{\prime}\left(V_{i}\right)$, different sets $S^{\star}\left(V_{i}\right)$ also correspond to disjoint sets $A_{i}$, centred at $W_{i}$, on the surface of the sphere $B$.

For each vector $\mathbf{e}$, there is an equatorial plane of the sphere $B$ at right angles to e. By (48) the point $W_{i}$ on the surface of $B$, where the normal is parallel to the normal $\mathbf{n}$ to $C_{1}$ at $T$, lies within a distance

$$
\leq \frac{26 \pi \delta c_{1} M}{c_{0} L|\mathbf{e}|}
$$

from the equatorial plane measured along the surface of $B$. As stated, the set $A_{i}$ is the intersection of the surface of $B$ with a ball of radius $\frac{1}{2} \sqrt{c_{0} \delta M}$, so it forms a two-dimensional ball in the spherical geometry of the surface of $B$, whose radius in spherical geometry is

$$
\leq \frac{\pi}{2} \cdot \sqrt{\frac{c_{0} \delta M}{4}} \leq \pi \sqrt{\frac{c_{0} \delta M}{16}} \cdot \frac{4 \sqrt{\delta c_{1} M}}{L|\mathbf{e}|}=\frac{\pi \delta c_{1} M}{L|\mathbf{e}|} \sqrt{\frac{c_{0}}{c_{1}}} \leq \frac{\pi \delta c_{1} M}{c_{0} L|\mathbf{e}|},
$$

by equation (4) and Lemma 4.1 .

Hence, each point of $A_{i}$ lies within a distance

$$
\leq \frac{26 \pi \delta c_{1} M}{c_{0} L|\mathbf{e}|}+\frac{\pi \delta c_{1} M}{c_{0} L|\mathbf{e}|}=\frac{27 \pi \delta c_{1} M}{c_{0} L|\mathbf{e}|}
$$

from the equatorial plane, measured along the surface of the sphere $B$.

We consider the "girdle" of one-dimensional boundary components $S^{\star}\left(V_{i}\right)$ which are parallel to the fixed vector $\mathbf{e}$. The components in the girdle satisfying (47) correspond to points $W_{i}$ and disjoint sets $A_{i}$ on the surface of $B$, such that every point of $A_{i}$ lies close to the equatorial plane perpendicular to e. The disjoint sets $A_{i}$ lie in an annulus whose volume in spherical geometry is at most

$$
\left(2 \pi c_{1} M\right)\left(\frac{54 \pi \delta c_{1} M}{c_{0} L|\mathbf{e}|}\right)=\frac{27(2 \pi)^{2} \delta\left(c_{1} M\right)^{2}}{c_{0} L|\mathbf{e}|} .
$$

By formula (49) the number of disjoint sets $A_{i}$ in the girdle is at most

$$
\frac{2^{2}}{\pi\left(c_{0} \delta M\right)} \cdot \frac{27(2 \pi)^{2} \delta\left(c_{1} M\right)^{2}}{c_{0} L|\mathbf{e}|}=\frac{27\left(4 \pi c_{1}\right)^{2} M}{\pi c_{0}^{2} L|\mathbf{e}|},
$$

so the boundary components $S^{\star}\left(V_{i}\right)$ in the girdle for which the number $l$ of 
points is in the range (47) contribute at most

$$
\frac{54 \pi\left(4 c_{1}\right)^{2} M}{c_{0}^{2}|\mathbf{e}|}
$$

integer points. The estimate (51) refers only to components in the girdle for which $l$ lies in the range (47).

We keep the condition (47), and sum over primitive integer vectors e. Since each component is a straight line segment lying within the strip $E$, by Lemma 4.1 we have

$$
L|\mathbf{e}| \leq(l-1)|\mathbf{e}| \leq 4 \sqrt{\delta c_{1} M} .
$$

We note that if two boundary components lie on the same line, then the vertices $V_{i}$ which label the boundary components $S^{\star}\left(V_{i}\right)$ must be different, so they are counted separately in this argument. We use Lemma 5.6 to sum over e, so that the number of points on one-dimensional boundary components with $l$ in the range (47) is at most

$$
\frac{54 \pi\left(4 c_{1}\right)^{2} M}{c_{0}^{2}} \cdot 2^{6}\left(\frac{4 \sqrt{\delta c_{1} M}}{L}\right)^{2}=\frac{2^{15} 3^{3} c_{1} \pi \delta\left(c_{1} M\right)^{2}}{c_{0}^{2} L^{2}} .
$$

Finally, we remove the condition (47) by summing $L$ through powers of 2 , and noting that

$$
\left(1+\frac{1}{2^{k}}+\frac{1}{4^{k}}+\frac{1}{8^{k}}+\cdots\right) \leq \frac{2^{k}}{2^{k}-1} \leq 2 .
$$

Hence the total number of integer points of $S(H)$ which lie on one-dimensional boundary components is at most

$$
\left(\frac{2^{16} 3^{3} c_{1} \pi}{c_{0}^{2}}\right) \delta\left(c_{1} M\right)^{2} \leq\left(\frac{2^{16} 3^{3} \pi c_{1}}{c_{0}^{2}}\right) \delta\left(c_{1} M\right)^{2}
$$

Lemma 6.2. The number of integer points lying on the plane boundary components is

$$
\leq 2^{19} \delta\left(c_{1} M\right)^{2} .
$$

Proof. For each plane boundary component, by (43) of Lemma 5.4, the integer points will all lie in a square of area

$$
400 \delta c_{1} M .
$$

The boundary components are convex sets lying on the convex hull $H$. Hence the convex hull of the plane boundary components, $H^{\prime}$ say, is a convex polyhedron contained within $H$, and the boundary components lie on the boundary planes of $H^{\prime}$. If the boundary of the convex polyhedron $H^{\prime}$ contains two parallel planes, then the convex body includes all points of the joins of any point on one plane with any point on the other plane. It follows that the outward normal vectors must be in opposite directions. Boundary 
components lie on boundary planes of $H^{\prime}$, so the direction of the outward normal determines the boundary plane.

Therefore, either the plane boundary components will all have different outward normal vectors $\mathbf{n}_{i}$, or some will share vectors and so form convex sets that all lie on the same plane. In the latter instance, these plane boundary components will all lie in an annulus as described in Lemma 4.2. As each component is convex in this annulus we can apply Lemma 4.5, and summing over all possible normal vectors gives the total number of integer points to be

$$
\leq 3 ! 2^{6} 3 \delta c_{1} M \sum \frac{1}{\left|\mathbf{n}_{i}\right|} .
$$

Applying similar logic to the former case yields

$$
\leq 3 ! 400 \delta c_{1} M \sum \frac{1}{\left|\mathbf{n}_{i}\right|}
$$

integer points. The constant in (55) is greater than that in (54) and for each $\mathbf{n}_{i}$ only one of the cases can occur. Hence we need only calculate the sum in (55). We note that the sum over all possible short normal vectors (length $\leq N$ ) will be greater than the sum over all possible long normal vectors (length $\geq N$ ) and so we consider twice the sum over the short normal vectors, giving

$$
\leq 2 \cdot 3 ! 400 \delta c_{1} M \sum_{1 \leq\left|\mathbf{n}_{i}\right| \leq N} \frac{1}{\left|\mathbf{n}_{i}\right|},
$$

where, by Theorem 3.4,

$$
N=2^{K}=\left(\frac{8 \pi}{27}\right)^{1 / 4}\left(c_{1} M\right)^{1 / 2} .
$$

Applying Lemma 5.6 yields

$$
\begin{aligned}
2 \cdot 3 ! 400 \delta c_{1} M \sum_{1 \leq\left|\mathbf{n}_{i}\right| \leq N} \frac{1}{\left|\mathbf{n}_{i}\right|} & \leq 2^{12} 3 \cdot 5^{2} \delta c_{1} M N^{2} \\
& \leq 2^{12} 3 \cdot 5^{2} \delta\left(c_{1} M\right)^{2} \leq 2^{19} \delta\left(c_{1} M\right)^{2},
\end{aligned}
$$

as required.

Lemma 6.3. The number of integer points on three-dimensional boundary components, when $\delta=\delta_{0}$, is estimated by

$$
\sum_{\operatorname{dim} S^{\star}\left(V_{i}\right)=3}\left|S^{\star}\left(V_{i}\right)\right| \leq 8 \cdot 24^{3 / 4}\left(2 c_{1} M\right)^{3 / 2} \leq 2^{9}\left(c_{1} M\right)^{3 / 2} .
$$


Proof. From (43), the three-dimensional boundary component $S^{\star}\left(V_{i}\right)$ will have a volume $\operatorname{Vol}\left(H_{i}\right)$, with

$$
\operatorname{Vol}\left(H_{i}\right) \leq \frac{52 \delta c_{1}}{c_{0}}\left(20 \sqrt{\delta c_{1} M}\right)^{2} .
$$

Since $\delta=\delta_{0}$ this gives a volume of at most $1 / 6$. Applying the minor arc case of Lemma 4.5 then gives

$$
K_{i} \leq 24 \mathrm{Vol}\left(H_{i}\right) \leq 4,
$$

where $K_{i}$ is the number of integer points contained in $S^{\star}\left(V_{i}\right)$. However, the existence of a three-dimensional $S^{\star}\left(V_{i}\right)$ in $S^{\prime}\left(V_{i}\right)$ requires that $K_{i} \geq 4$, and so if we consider $\delta=\delta_{0}$, then $K_{i}$, the number of integer points in the boundary component, is exactly 4 . The number of vertices of the convex hull is

$$
\leq 2 \cdot 24^{3 / 4}\left(2 c_{1} M\right)^{3 / 2},
$$

by (22) in Theorem 3.6. Hence, when $\delta=\delta_{0}$, the total number of integer points in the three-dimensional boundary components is

$$
\leq 8 \cdot 24^{3 / 4}\left(2 c_{1} M\right)^{3 / 2} \leq 2^{9}\left(c_{1} M\right)^{3 / 2} .
$$

We now collect together the terms (22), (46), (53), (56) and (36) to obtain an upper bound for the total number of integer points contributed from the $j$-dimensional extended vertex components, $0 \leq j \leq 3$, along with the internal integer points, when $\delta \leq \delta_{0}$. This gives

$$
\begin{aligned}
& \leq\left(\frac{c_{1}}{c_{0}}\right)\left(\left(2^{7}+2^{9}\right)\left(c_{1} M\right)^{3 / 2}+\left(2^{19}+2^{16} 3^{3} \pi+2^{8}\right) \delta_{0}\left(c_{1} M\right)^{2}\right) \\
& \leq\left(\frac{c_{1}}{c_{0}}\right)\left(2^{10}\left(c_{1} M\right)^{3 / 2}+2^{23} \delta_{0}\left(c_{1} M\right)^{2}\right) .
\end{aligned}
$$

This result is valid for a shell of thickness $\delta=\delta_{0}$ and consists of terms independent of $\delta$ (degree zero), and those with a factor of $\delta$ (degree one).

We cover the shell $E$ of all extended vertex components, bounded internally by $C_{0}$ and externally by $C_{1}$, by $R$ thinner concentric shells $E_{1}, \ldots, E_{R}$ of thickness $\delta_{0}$. The distance between $C_{1}$ and $C_{0}$ along any inward normal vector to these two surfaces is $2 \delta$. Hence we choose $R$ to be the smallest such integer with

$$
R \delta_{0} \geq 2 \delta, \quad(R-1) \delta_{0}<2 \delta,
$$

so that

$$
R<2 \delta / \delta_{0}+1 .
$$

The shell $E_{r}$ consists of the points on some inward normal whose distance $l$ from the surface $C_{1}$ lies in the range

$$
(r-1) \delta_{0} \leq l \leq r \delta_{0} .
$$


When we replace $\delta$ with $r \delta_{0}$ in Lemma 2.1, we see that each shell $E_{r}$ will satisfy the Curvature Condition, so that any plane sectional curve of $E_{r}$ will lie in the range

$$
c_{0} M \leq \varrho \leq c_{1} M .
$$

Therefore, expression (59) gives a uniform upper bound for the number of integer points contributed by any shell $E_{r}$. We note that

$$
\delta_{0} \sqrt{c_{1} M} \leq \frac{c_{1}}{c_{0}} \cdot \frac{1}{2^{8}}
$$

and

$$
\left(\delta_{0} \sqrt{c_{1} M}\right)^{-1} \leq \frac{c_{1}}{c_{0}} 2^{9} .
$$

THEOREM 6.4. The number of integer points lying on or within a short distance $\delta$ from a convex closed surface that is contained in a sphere of radius $c_{1} M$ in three-dimensional Euclidean space is

$$
\leq\left(\frac{c_{1}}{c_{0}}\right)^{2} 2^{16}\left(\left(c_{1} M\right)^{3 / 2}+2^{9} \delta\left(c_{1} M\right)^{2}\right) .
$$

Proof. We multiply the upper bound (59) by the maximum number of shells given by (60). This yields

$$
\left(\frac{2 \delta}{\delta_{0}}+1\right) \frac{c_{1}}{c_{0}}\left(2^{10}\left(c_{1} M\right)^{3 / 2}+2^{23} \delta_{0}\left(c_{1} M\right)^{2}\right) .
$$

Simplifying using (61) and (62) and combining terms we have at most

$$
\left(\frac{c_{1}}{c_{0}}\right)^{2} 2^{16}\left(\left(c_{1} M\right)^{3 / 2}+2^{9} \delta\left(c_{1} M\right)^{2}\right)
$$

integer points.

This paper forms part of INTAS research project 03-51-5070 on analytic and combinatorial methods in number theory and geometry as well as part of my PhD thesis in the University of Wales.

I would like to thank my supervisor Professor M. N. Huxley for all his invaluable support and guidance in this problem.

\section{References}

[1] G. E. Andrews, An asymptotic expression for the number of solutions of a general class of diophantine equations, Trans. Amer. Math. Soc. 99 (1961), 272-277.

[2] - A lower bound for the volume of strictly convex bodies with many boundary lattice points, ibid. 106 (1963), 270-279.

[3] M. Beck, J. A. De Loera, M. Develin, J. Pfeifle and R. P. Stanley, Coefficients and roots of Ehrhart polynomials, in: Contemp. Math. 374, Amer. Math. Soc., 2005, $15-36$.

[4] M. Beck and S. Robins, Computing the Continuous Discretely, Springer, 2007. 
[5] M. Branton et P. Sargos, Points entiers au voisinage d'une courbe plane à très faible courbure, Bull. Sci. Math. 118 (1994), 15-28.

[6] M. Filaseta and O. Trifonov, The distribution of fractional parts with applications to gap results in number theory, Proc. London Math. Soc. (3) 73 (1996), 241-278.

[7] P. M. Gruber and C. G. Lekkerkerker, Geometry of Numbers, 2nd ed., North-Holland Math. Library 37, North-Holland, 1987.

[8] E. Hlawka, Integrale auf konvexen Körpern, I, II, Monatsh. Math. 54 (1950), 1-36, $81-99$.

[9] M. N. Huxley, Area, Lattice Points and Exponential Sums, Oxford Univ. Press, 1996.

[10] - The integer points close to a curve III, in: Number Theory in Progress (Zakopane-Kościelisko, 1997), K. Győry et al. (eds.), de Gruyter, 1999, Vol. II, 911-940.

[11] M. N. Huxley et P. Sargos, Points entiers au voisinage d'une courbe plane de classe $C^{n}, I I$, Funct. Approx. Comment. Math. 35 (2006), 91-115.

[12] V. Jarník, Über die Gitterpunkte auf konvexen Kurven, Math. Z. 24 (1925), 500-518.

[13] E. Krätzel, Analytische Funktionen in der Zahlentheorie, Teubner, 2000.

[14] M. C. Lettington, PhD thesis, Cardiff Univ., 2008.

[15] - Integer points close to convex hypersurfaces, in preparation.

[16] P. McMullen, The maximum numbers of faces of a convex polytope, Mathematika 17 (1970), 179-184.

[17] P. McMullen and G. C. Shephard, Convex Polytopes and the Upper Bound Conjecture, London Math. Soc. Lecture Note Ser. 3, Cambridge Univ. Press, 1971.

[18] W. Müller, Lattice points in large convex bodies, Monatsh. Math. 128 (1999), 315330.

[19] D. M. Y. Sommerville, An Introduction to the Geometry of n Dimensions, Methuen, London, 1929.

School of Mathematics

Cardiff University

P.O. Box 926

Cardiff CF24 4YH, UK

E-mail: LettingtonMC@cf.ac.uk

matt.lettington@sky.com

Received on 29.1.2008

and in revised form on 2.1.2009 